\title{
Research on Experiments of Perfect Shuffle Implementation Using Micro-Optical Array Element*
}

\author{
Ping $\mathrm{Xu}^{* *} \quad$ Haixuan Huang Zelin Yan Lili Wan Shanshan Tu \\ College of Engineering and Technology, ShenZhen University Guangdong, Shenzhen 518060, \\ P.R.China
}

\begin{abstract}
In this paper, a binary optical array element which can realize 8-channel PS transformation is fabricated with very large scale integration (VLSI), stepping photolithography and reactive ion etching (RIE). Using the element, we perform experiments of the PS transformation, tests and analysis. The efficiency and cross talk of each channel are measured when only one channel has been reserved while other channels have been closed. The result indicates that the method proposed to realize the PS transformation by using the micro-optical array element agrees well with theoretical expectation. This success of the experiment lays a good foundation for us to do further research on optical transformation through multilevel interconnection.
\end{abstract}

KEYWORDS: Binary optics; perfect shuffle; reactive ion etching; array element; diffractive efficiency;

\section{INTRODUCTION}

With the development of Information Superhighway and burgeoning digital optical computer, more and more attention has been paid to free-spatial optical interconnection (FOSI). Hereinto, the PS transformation, as the basic transformation network, can implement arbitrary transformation through multilevel interconnection and combination. ${ }^{1}$ The author of the paper has ever presented a new method to realize the PS transformation with micro-blazed grating array. ${ }^{2-3}$ Using only one element, we can easily realized one and two-dimensional PS transformation, and their inverse transformations. Due to the application of deep relief and microstructure, it is rather easy to realize micromation and multilevel interconnection of elements, which can meet the needs of realizing arbitrary transformation in optical interconnection. Therefore, it has an important academic significance and applied value. Based on the design parameters provided by Reference 3, a binary optical array element that can realize 8-channel PS transformation is fabricated with VLSI, stepping photolithography and RIE. Tests and experiments reveal that, using only one array element, one-dimensional PS transformation can be easily realized, instead of using intricate optical device or spatial filtering to realize signal division, interleaving and order reversing. The output image is erect, and the empty-occupation rate can be adjusted arbitrarily. The element works with relatively high light utilization efficiency and low channel cross talk, and its average efficiency reaches $84.58 \%$.

*This work is supported by the National Nature Science Foundation of China(No.60178023) and the Science \& Technology Development Fund of Shenzhen.

*** Contact author, E-mail xuping@szu.edu.cn Tel.(0755)26557246 


\section{THEORY}

Perfect shuffle refers to a transformation that a group of elements (signal) are made to go halves, intervened, and inserted in specified order, as shown in Fig.1. Assume the number of elements input is $\mathrm{N}$, then according to a specified rule, elements in arbitrary order can be transformed to original tactic order after $3 \log _{2} \mathrm{~N}$ times' transformation. Thus, it can be applied as a basic interconnected element in optical computing or switching.

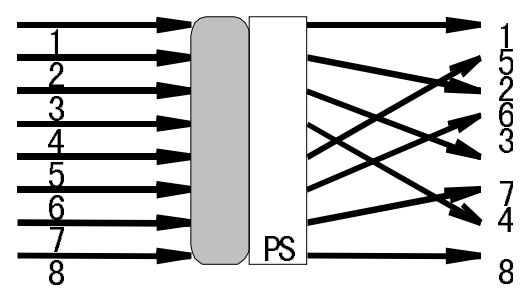

Fg.1 PS transformation

Based on the rule of perfect shuffle and the working principle of blazed grating, a PS plate is designed and fabricated with the technology which we have developed to fabricate deep relief binary optical elements ${ }^{4--6}$. The main idea for design is as follows: Light can be output in different directions through gratings with different spatial frequencies. Gratings are combined reasonably to control a light beam to accurately output abiding by the rules of the PS transformation. Therefore, an array element composed of micro-blazed gratings with different spatial frequencies is designed to realize the PS transformation. Due to the use of structural combination of the deep relief blazed gratings, the light ultization efficiency of the element is very high. Correlative references delivered by the author of this paper can be referred to with regard to getting information about theoretical analysis and design of perfect shuffle implementation using the micro-optical array element. ${ }^{3}$

\section{EXPERIMENTAL SETUPAND RESULTS}

Experiments are performed with a He-Ne laser served as light source. A laser beam is expanded and collimated when turned into parallel light, and then it is applied to the 8-channel micro-optical array element through a diaphragm. The experimental setup is shown in Fig.2. An optical power meter made by COHERENT Corporation in USA is used for detecting laser power. (Its working spectrum ranges from $400 \mathrm{~nm}$ to $1064 \mathrm{~nm}$, and the detecting power ranges from $10 \mathrm{nW}$ to $50 \mathrm{~mW}$ )

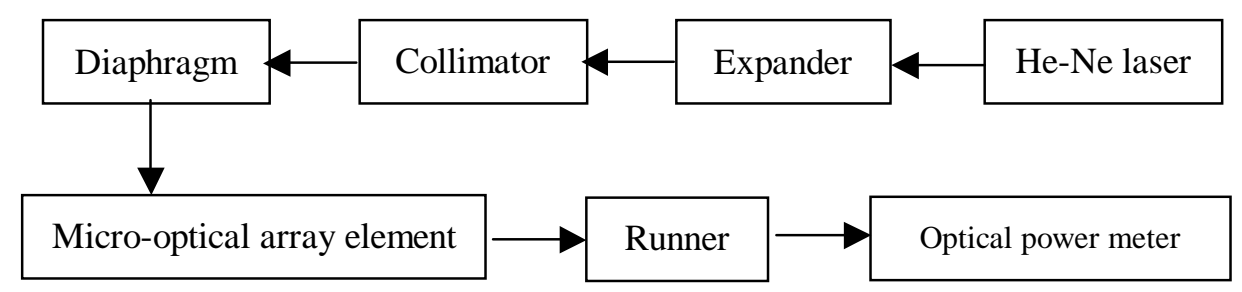

Fig.2 Experimental setup for performing the PS transformation

The input signal designed and fabricated for the experiment is shown in Fig.3. Let the contents of the input signal 
be a set of integers $\{1,2,3,4,5,6,7,8\}$, which are arranged in two rows: the upper row and the lower row. Hereinto, the upper row is used for comparison instead of attending the transformation, and the lower row is the real input signal. The input signal is applied to the PS plate, which consists of 8-channel micro-optical array element. The experimental result is displayed in Fig.4, after the illumination of parallel light and the transformation operated by the element. As it is shown in Fig.4, the upper row is the projection of input signal that does not attend the transformation, and the lower row is the result of input signal after transformation.

The serial numbers of the input channels are $I_{1}, I_{2}, I_{3}, I_{4}, I_{5}, I_{6}, I_{7}, I_{8}$, and the corresponding sequence of the input signal is $\{1,2,3,4,5,6,7,8\}$. What is apparently displayed in the lower row in Fig.4 is that ,the sequence of the output signal is $\{1,5,2,6,3,7,4,8\}$. That is to say, after the transformation operated by the element, the input signal sequence $\{1,2,3,4,5,6,7,8\}$ is transformed into an output sequence $\{1,5,2,6,3,7,4,8\}$. Apparently, such a sequence transformation agrees well with the rule of perfect shuffle shown in Fig.1.

Results from the experiment suggest that the optical PS transformation can be successfully realized by using only one element.

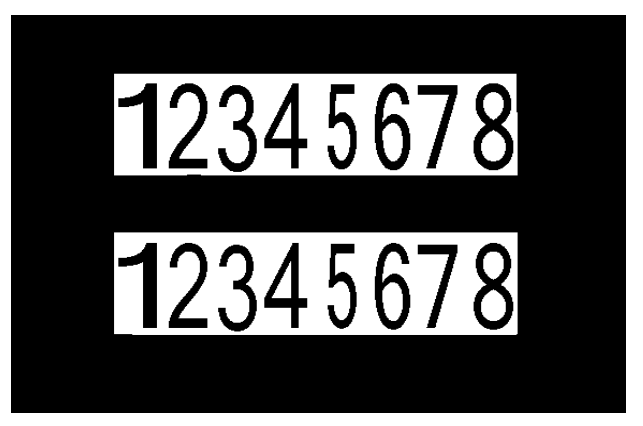

Fig.3 The input signal

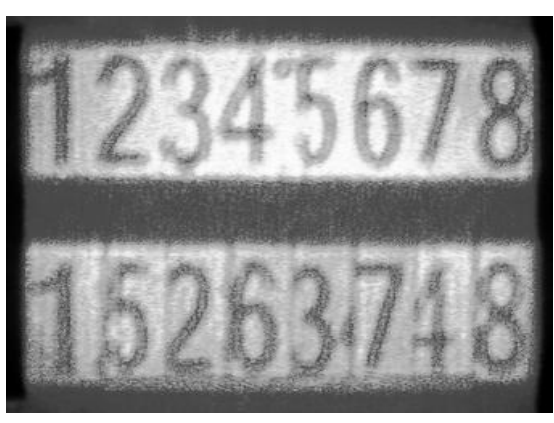

Fig.4 The output signal

\section{TESTS AND ANALYSIS OF THE EFFICIENCY AND CROSS TALK}

To test the efficiency and cross talk of each channel, a structure of runner is specially designed, as it is shown in Fig5.

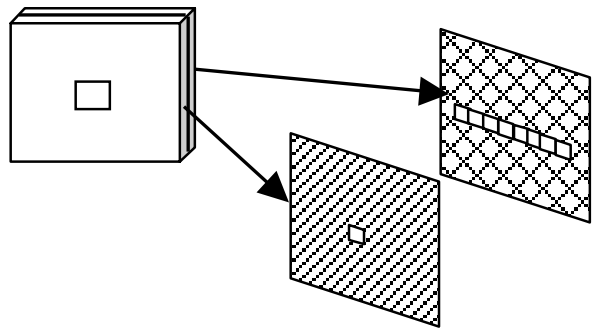

Fig.5 Structure of the runner

With the devices shown in Fig.2 and Fig.5, the efficiency and cross talk of each channel is measured when only one channel has been reserved while other channels have been closed. A screen with a rectangle aperture about a channel-width is attached to the 8-channel micro-optical array element. As a result, the laser beam is limited to go through only one channel. An optical power meter can be used to measure light power of the input channel. Next, the runner is dragged to measure respectively the light power of each output channel. The metrical result is shown in Tab. 1 
Tab.1 An analysis of each channel's diffractive efficiency and cross talk (Unit:\%)

\begin{tabular}{|c|c|c|c|c|c|c|c|c|c|}
\multicolumn{2}{|c|}{ Output } & $\mathrm{O}_{1}$ & $\mathrm{O}_{2}$ & $\mathrm{O}_{3}$ & $\mathrm{O}_{4}$ & $\mathrm{O}_{5}$ & $\mathrm{O}_{6}$ & $\mathrm{O}_{7}$ & $\mathrm{O}_{8}$ \\
\cline { 2 - 11 } $\mathrm{Input}$ & 1 & 5 & 2 & 6 & 3 & 7 & 4 & 8 \\
\hline $\mathrm{I}_{1}$ & 1 & 95.30 & & & & & & & \\
\hline $\mathrm{I}_{2}$ & 2 & 1.42 & 6.59 & 78.57 & 1.89 & 0.50 & 0.47 & 1.47 & 0.36 \\
\hline $\mathrm{I}_{3}$ & 3 & 7.63 & 0.94 & 3.16 & 1.90 & 79.03 & 4.14 & 0.52 & 0.57 \\
\hline $\mathrm{I}_{4}$ & 4 & 3.21 & 0.44 & 0.65 & 0.90 & 0.68 & 2.38 & 88.95 & 1.09 \\
\hline $\mathrm{I}_{5}$ & 5 & 1.05 & 86.86 & 1.70 & 0.83 & 5.23 & 0.55 & 0.40 & 2.20 \\
\hline $\mathrm{I}_{6}$ & 6 & 0.67 & 1.61 & 2.08 & 73.86 & 2.94 & 3.99 & 0.64 & 7.61 \\
\hline $\mathrm{I}_{7}$ & 7 & 0.62 & 0.80 & 0.59 & 0.81 & 2.47 & 80.00 & 4.24 & 1.16 \\
\hline $\mathrm{I}_{8}$ & 8 & & & & & & & & 94.05 \\
\hline
\end{tabular}

Now we perform a brief analysis of the data in Tab.1.

Firstly, each channel has a relatively high output of light power, abiding by the rule of perfect shuffle. Take 4th input channel $\mathrm{I}_{4}$ for example. The input power is mainly output to $\mathrm{O}_{7}$, reaching $88.95 \%$. The other channels can be analyzed similarly. It is concluded that the element works with relatively high light utilization efficiency and its average transpositional efficiency reaches $84.58 \%$.

Secondly, the power output to other channels that the input signal should not go through is rather low; that is to say, the element has very low cross talk to other channels. The same conclusion comes out, based on the analysis of other input channels. Consequently, the output signals have little influence on each other.

Of course, the diffractive efficiency obtained from the experiment is lower than our theoretical value. The main reasons may lie in errors during the fabrication of the element and errors in experimental tests, such as instability of laser's output power, errors of the optical path collimation, errors of observation when dragging the runner. These factors are to be improved further.

\section{CONCLUSIONS}

In this paper, the binary optical array element which can realize 8-channel PS transformation is fabricated with VLSI, stepping photolithography and RIE. Using the element, we perform experiments of the PS transformation, tests and analysis. Results from the experiments reveal that, one-dimensional PS transformation can be easily realized by using only one array element, instead of using intricate optical device or spatial filtering to realize signal division, interleaving and order reversing. The average efficiency reaches $84 \%$. The output image is erect, and the cross talk is low. The result indicates that, the method proposed to realize the PS transformation by using micro-optical array element agrees well with theoretical expectation ${ }^{2-3}$. This success of the experiments lays a good foundation for us to do further research on the optical transformation through multilevel interconnection.

\section{REFERENCES}

[1] A.W.Lohmann, What Classical Optics Can Do for the Digital Optical Computer, Appl.Opt. Vol.25, No.10, pp1543-1549,1986. 
[2] Ping $\mathrm{Xu}$, etc., Optical Perfect Shuffle Interconnection Using Computer-Generated Blazed Grating Array , OPTICAL REVIEW VOL.2, No.5(1995)362-365

[3] Ping $\mathrm{Xu}$, etc., Theoretical Analysis And Design Of Perfect Shuffle Implementation Using Micro-optical Array Element , Proc.SPIE Vol 5636.52,2004

[4] Ping Xu, etc., Deep Etch Binary Optics Element, Acta Optica Sinica (Chinese), Vol.16, No.12, 1996

[5] Ping Xu, etc., The even device fabricated by the deep etched binary optics technogy for the exposure system of the quasi-molecule laser, Science In China (Series E)

Vol.45,No.1,1-9,2002

[6] Ping Xu, etc., Fabrication errors analysis and simulation of binary optical element, Acta Optica Sinica (Chinese), Vol.16, No.5, 1996 